PROCEEDINGS OF THE

AMERICAN MATHEMATICAL SOCIETY

Volume 132, Number 6, Pages 1849-1853

S 0002-9939(03)07286-1

Article electronically published on November 7, 2003

\title{
PRODUCTS OF SPAN ZERO CONTINUA AND THE FIXED POINT PROPERTY
}

\author{
M. M. MARSH
}

(Communicated by Alan Dow)

\begin{abstract}
We prove that products of surjective mappings from continua onto span zero continua are universal. It follows that products of span zero continua have the fixed point property.
\end{abstract}

A continuum is a compact, connected metric space. By a map or mapping we mean a continuous function. A continuum $X$ has the fixed point property (fpp) if each self-map of $X$ has a fixed point.

The fixed point property for continua is not, in general, preserved under Cartesian products. In fact, there are examples where

(1) $X$ is a polyhedron with the fpp, $I$ is the unit interval, and $X \times I$ does not have the fpp (see [11]),

(2) $X$ and $Y$ are manifolds with the fpp, and $X \times Y$ does not have the fpp (see 9]).

See R. F. Brown's article [1] for some history and discussion of this topic.

The Brouwer Fixed Point Theorem established that the finite product of arcs has the fixed point property. There have been many generalizations of Brouwer's theorem. In particular, in 1956 Eldon Dyer [5] showed that arbitrary products of arc-like (chainable) continua have the fpp. Holsztyński [8] generalized Dyer's result in 1968 by showing that products of mappings of continua onto products of arc-like continua are universal. Since every arc-like continuum has span zero, we generalize both of these results by showing that products of mappings of continua onto span zero continua are universal. It follows that products of span zero continua have the fpp. It is not known if span zero continua are arc-like. This question is a classic problem in the study of continua (see [3, Prob. 8]).

Holsztyński called a mapping $f: X \rightarrow Y$ between topological spaces universal provided that whenever $g: X \rightarrow Y$ is a mapping, it follows that $f$ and $g$ have a coincidence; that is, there is a point $x \in X$ such that $f(x)=g(x)$. It is easy to see that if $f: X \rightarrow Y$ is universal, then $f$ is surjective and $Y$ has the fpp.

A mapping $f: X \rightarrow Y$ is an $\epsilon$-map if for $y \in Y$, $\operatorname{diam} f^{-1}(y)<\epsilon$. A continuum $X$ is arc-like (tree-like) if for each $\epsilon>0$, there exists an $\epsilon$-map of $X$ onto an arc (a tree).

For a continuum $X$, let $\pi_{1}$ and $\pi_{2}$ denote the coordinate projection maps of $X \times X$ onto $X$. The continuum $X$ has span zero (has semispan zero), denoted

Received by the editors October 2, 2002 and, in revised form, February 21, 2003.

2000 Mathematics Subject Classification. Primary 54H25, 54B10; Secondary 54F15, 54C10.

(C)2003 American Mathematical Society 
by $\sigma(X)=0\left(\sigma_{0}(X)=0\right)$, if whenever $Z$ is a continuum in $X \times X$ such that $\pi_{1}(Z)=\pi_{2}(Z)\left(\pi_{2}(Z) \subseteq \pi_{1}(Z)\right)$, then $Z$ intersects the diagonal in $X \times X$. The two types of span zero become surjective span zero if we require that $\pi_{1}(Z)=X$ in the definitions above. The notations for surjective span zero and surjective semispan zero are, respectively, $\sigma^{*}(X)=0$ and $\sigma_{0}^{*}(X)=0$. The results in this paper are for continua $X$ such that $\sigma_{0}^{*}(X)=0$. James F. Davis [4] has shown that $\sigma_{0}(X)=0$ is equivalent to $\sigma(X)=0$, and it is easy to see that $\sigma_{0}(X)=0$ implies $\sigma_{0}^{*}(X)=0$. Hence, our results hold for continua possessing any of these forms of span zero.

Let $X$ be a connected space with subsets $F, A$, and $B$, and suppose that $A$ and $B$ are closed and disjoint. We say that $F$ separates $X$ between $A$ and $B$ if $X-F$ is not connected between $A$ and $B$. That is, if there exist mutually separated sets $M$ and $N$ such that $X-F=M \cup N, A \subseteq M$, and $B \subseteq N$. We point out that if $F$ separates $X$ between $A$ and $B$, then $F$ is disjoint from $A \cup B$. We say that the closed set $F$ weakly cuts $A$ from $B$ in $X$ (or $F$ weakly cuts $X$ between $A$ and $B$ ) if each closed connected set in $X$ that intersects both $A$ and $B$ must also intersect $F$. The closed set $F$ need not be disjoint from $A \cup B$ to weakly cut $A$ from $B$.

If $F$ is closed and separates $X$ between $A$ and $B$, then there exist closed sets $P$ and $Q$ such that $X=P \cup Q, F=P \cap Q, A \subseteq P$, and $B \subseteq Q$ (see [10, p. 155]). Furthermore, in this case, if we take $U=P-F$ and $V=Q-F$, we have a partition of $X$ between $A$ and $B$ as defined by Holsztyński in [7, 8. That is, $U$ and $V$ are open sets in $X$ such that $X-F=U \cup V, A \subseteq U, B \subseteq V$, and $U \cap V=\emptyset$. In fact, it is easy to see that when $F$ is closed, $F$ partitions $X$ between $A$ and $B$ if and only if $F$ separates $X$ between $A$ and $B$.

The proof of our main theorem follows methods used by Holsztyński in [7], [8]. A basic idea underlying the proof also involves weak cutting between certain continua in spaces that resemble products or cones. This notion has previously been used by the author [13] to obtain fixed point results. Recently, J. Bustamante, R. Escobedo, and F. Macías (see [2]), and R. Escobedo, M. de J. López, and S. Macías (see [6]) used this technique in a clever way to obtain similar results in hyperspaces. R. Escobedo recently announced obtaining the result that the product of two mappings from continua onto span zero continua must be universal.

Lemma 1. Suppose $F$ is a closed set in the continuum $X$ that weakly cuts $X$ between the continua $A$ and $B$. If $U$ is an open set containing $F$, then there is a closed set $E \subseteq U$ such that $E$ separates $X$ between $A$ and $B$.

Proof. Suppose one of $A$ or $B$ is a subset of $U$. Suppose $A \subseteq U$. Let $V$ be an open set such that $A \subseteq V \subseteq \bar{V} \subseteq U$ and $\bar{V} \cap B=\emptyset$. Then the boundary of $V$ is a closed set in $U$ that separates $X$ between $A$ and $B$. Thus, we may assume that neither $A$ nor $B$ is a subset of $U$.

First we show that $U-(A \cup B)$ separates $X$ between $A$ and $B$. Let $A^{\prime}=\{x \in$ $X-U \mid$ there exists a continuum $C \subseteq X-U$ such that $x \in C$ and $C \cap(A-U) \neq \emptyset\}$. Let $B^{\prime}=\{x \in X-U \mid$ there exists a continuum $C \subseteq X-U$ such that $x \in$ $C$ and $C \cap(B-U) \neq \emptyset\}$. Clearly, $A^{\prime}$ and $B^{\prime}$ are closed, $A-U \subseteq A^{\prime}$, and $B-U \subseteq B^{\prime}$. Since $F$ weakly cuts $X$ between $A$ and $B$, no subcontinuum of $X-U$ intersects both $A^{\prime}$ and $B^{\prime}$. By the Wire Cutting Theorem (see [14, Th. 44, p. 15]), $U$ separates $X$ between $A^{\prime}$ and $B^{\prime}$, and hence between $A-U$ and $B-U$. Since $A \cap B=\emptyset$, it follows that $U-(A \cup B)$ separates $X$ between $A$ and $B$.

By [10, Th. 3, p. 155], it follows that $U-(A \cup B)$ contains a closed set $E$ that separates $X$ between $A$ and $B$. 
The author wishes to thank Eldon Vought for providing the proof of the lemma above, which is shorter than the author's original proof.

Theorem 1. For $1 \leq i \leq n$, let $X_{i}$ and $M_{i}$ be continua. Suppose that for each $1 \leq i \leq n, \sigma_{0}^{*}\left(M_{i}\right)=0$ and $f_{i}: X_{i} \rightarrow M_{i}$ is a surjective mapping. Then the product mapping

$$
\prod_{i=1}^{n} f_{i}: \prod_{i=1}^{n} X_{i} \rightarrow \prod_{i=1}^{n} M_{i}
$$

is universal.

Proof. Let $f=\prod_{i=1}^{n} f_{i}, X=\prod_{i=1}^{n} X_{i}$, and $M=\prod_{i=1}^{n} M_{i}$. For each $1 \leq i \leq n$, $M_{i}$ is atriodic, tree-like (see [15]), and thus, by [16], each $M_{i}$ is irreducible between some two points, say $a_{-i}$ and $a_{i}$. Let $I=[-1,1]$ and $I^{n}=\prod_{i=1}^{n} I$. It will be convenient to let $\pi_{i}: Y \rightarrow Y_{i}$ denote coordinate projection for $Y \in\left\{X, M, I^{n}\right\}$ and $1 \leq i \leq n$.

Let $g: X \rightarrow M$ be a mapping. We will show that $f$ and $g$ have a coincidence.

For $1 \leq i \leq n$, let $F_{i}=\left\{x \in X \mid \pi_{i} f(x)=\pi_{i} g(x)\right\}$. We note that since $f$ is a product mapping, $\pi_{i} \circ f=f_{i} \circ \pi_{i}$ for $1 \leq i \leq n$. Since $\sigma_{0}^{*}\left(M_{i}\right)=0$, it is well known that each mapping of a continuum onto $M_{i}$ is universal. In fact, $\sigma_{0}^{*}\left(M_{i}\right)=0$ is characterized by $M_{i}$ admitting only surjective maps that are universal (see [12]). It follows that $F_{i} \neq \emptyset$ for each $1 \leq i \leq n$. Also, clearly each $F_{i}$ is a closed set.

For $1 \leq i \leq n$, let $b_{-i} \in f_{i}^{-1}\left(a_{-i}\right), b_{i} \in f_{i}^{-1}\left(a_{i}\right), B_{-i}=\pi_{i}^{-1}\left(b_{-i}\right)$, and $B_{i}=$ $\pi_{i}^{-1}\left(b_{i}\right)$. We refer to $B_{-i}$ and $B_{i}$ as a pair of opposite sides of $X$.

We now show that $F_{i}$ weakly cuts $B_{-i}$ from $B_{i}$ in $X$ for each $1 \leq i \leq n$. Fix $i$ with $1 \leq i \leq n$. Suppose that $C$ is a continuum in $X$ that intersects both $B_{-i}$ and $B_{i}$. Then $\pi_{i} f(C)=M_{i}$ since $M_{i}$ is irreducible between $a_{-i}$ and $a_{i}$. Thus, $\left.\pi_{i} f\right|_{C}: C \rightarrow M_{i}$ is universal and has a coincidence with the map $\left.\pi_{i} g\right|_{C}: C \rightarrow M_{i}$. So, $C \cap F_{i} \neq \emptyset$. Hence, $F_{i}$ weakly cuts $B_{-i}$ from $B_{i}$ in $X$.

If $\bigcap_{i=1}^{n} F_{i} \neq \emptyset$, then there is a point $x \in X$ such that for each $1 \leq i \leq n$, $\pi_{i} f(x)=\pi_{i} g(x)$. So, $f(x)=g(x)$ and we are done. Therefore, we assume that $\bigcap_{i=1}^{n} F_{i}=\emptyset$.

For $1 \leq i \leq n$, there exist an open set $U_{i}$ in $X$ such that $F_{i} \subseteq U_{i}$, and $\bigcap_{i=1}^{n} U_{i}=$ $\emptyset$. By Lemma 1 , for each $1 \leq i \leq n$, there exists a closed set $E_{i} \subseteq U_{i}$ such that $E_{i}$ separates $X$ between $B_{-i}$ and $B_{i}$ and $E_{i} \cap\left(B_{-i} \cup B_{i}\right)=\emptyset$. Also, $\bigcap_{i=1}^{n} E_{i}=\emptyset$. Finally, for $1 \leq i \leq n$, we let $G_{i}$ be open in $X$ with $E_{i} \subseteq G_{i}, G_{i} \cap\left(B_{-i} \cup B_{i}\right)=\emptyset$, and $\bigcap_{i=1}^{n} G_{i}=\emptyset$.

For $1 \leq i \leq n$, let $H_{-i}$ and $H_{i}$ be open sets in $X$ such that $H_{-i} \cap E_{i}=H_{i} \cap E_{i}=$ $H_{-i} \cap H_{i}=\emptyset, B_{-i} \subseteq H_{-i}-G_{i}, B_{i} \subseteq H_{i}-G_{i}$, and $H_{-i} \cup H_{i}=X-E_{i}$.

For $1 \leq i \leq n$, let $h_{i}: X_{i} \rightarrow I$ be a mapping where $h_{i}^{-1}(-1)=\left\{b_{-i}\right\}$ and $h_{i}^{-1}(1)=\left\{b_{i}\right\}$. By Holsztyński [8, Th. 2.5], $h=\prod_{i=1}^{n} h_{i}: X \rightarrow I^{n}$ is universal.

For $1 \leq i \leq n$, let $I_{-i}^{n}=\pi_{i}^{-1}(-1)$ and $I_{i}^{n}=\pi_{i}^{-1}(1)$ be opposite sides of $I^{n}$. Once again, since $h$ is a product mapping, we have that $\pi_{i} \circ h=h_{i} \circ \pi_{i}$ for $1 \leq i \leq n$. It follows that for each $1 \leq i \leq n, h^{-1}\left(I_{-i}^{n}\right)=B_{-i} \subseteq H_{-i}$ and $h^{-1}\left(I_{i}^{n}\right)=B_{i} \subseteq H_{i}$. For $1 \leq i \leq n$, the set $H_{i}-G_{i}$ is closed in $X$ since $H_{i}-G_{i}=X-\left(G_{i} \cup H_{-i}\right)$ is closed in $X$. Similarly, $H_{-i}-G_{i}$ is closed in $X$. So, for $1 \leq i \leq n$, define mappings $k_{i}: X \rightarrow I$ such that $k_{i}(x)=1$ for $x \in H_{-i}-G_{i}$ and $k_{i}(x)=-1$ for $x \in H_{i}-G_{i}$. Let $k: X \rightarrow I^{n}$ be defined by $k(x)=\left(k_{1}(x), \ldots, k_{n}(x)\right)$. 
We get that

$$
\begin{aligned}
\bigcup_{i=1}^{n}\left(\left(H_{-i}-G_{i}\right) \cup\left(H_{i}-G_{i}\right)\right) & =\bigcup_{i=1}^{n}\left(\left(H_{-i} \cup H_{i}\right)-G_{i}\right) \\
& =\bigcup_{i=1}^{n}\left(\left(X-E_{i}\right)-G_{i}\right) \\
& =\bigcup_{i=1}^{n}\left(X-G_{i}\right) \\
& =X-\bigcap_{i=1}^{n} G_{i}=X .
\end{aligned}
$$

Recall that $h: X \rightarrow I^{n}$ is universal. So, $h$ and $k$ have a coincidence. Let $x \in X$ be such that $h(x)=k(x)$. From the equalities above, we have that $x$ is in $\left(H_{-i}-G_{i}\right) \cup\left(H_{i}-G_{i}\right)$ for some $1 \leq i \leq n$. Suppose, without loss of generality, that $x \in H_{i}-G_{i}$ for some $1 \leq i \leq n$. Thus, $k(x)$ has $i$ th coordinate $k_{i}(x)=-1$. So, the $i$ th coordinate of $h(x)$ is also -1 . That is, $\pi_{i} h(x)=h_{i} \pi_{i}(x)=-1$; so $x \in h^{-1}\left(I_{-i}^{n}\right)=B_{-i} \subseteq H_{-i}-G_{i}$. But $\left(H_{-i}-G_{i}\right) \cap\left(H_{i}-G_{i}\right)=\emptyset$; so we have a contradiction.

Hence, $\bigcap_{i=1}^{n} F_{i} \neq \emptyset$ and it follows that $f$ and $g$ have a coincidence.

Corollary 1. Let $\left\{M_{\alpha} \mid \alpha \in \Gamma\right\}$ and $\left\{X_{\alpha} \mid \alpha \in \Gamma\right\}$ be families of continua such that $\sigma_{0}^{*}\left(M_{\alpha}\right)=0$ for each $\alpha \in \Gamma$. Let $\left\{f_{\alpha}: X_{\alpha} \rightarrow M_{\alpha} \mid \alpha \in \Gamma\right\}$ be a family of surjective mappings. Then

$$
\prod_{\alpha \in \Gamma} f_{\alpha}: \prod_{\alpha \in \Gamma} X_{\alpha} \rightarrow \prod_{\alpha \in \Gamma} M_{\alpha}
$$

is universal.

Proof. By Theorem 1, each finite product of mappings on a finite subfamily of $\left\{f_{\alpha}: X_{\alpha} \rightarrow M_{\alpha} \mid \alpha \in \Gamma\right\}$ is universal. So, by Theorem 3.1 in [8], the result follows.

Corollary 2. Let $\left\{M_{\alpha} \mid \alpha \in \Gamma\right\}$ be a family of continua such that $\sigma_{0}^{*}\left(M_{\alpha}\right)=0$ for each $\alpha \in \Gamma$. The identity mapping of $\prod_{\alpha \in \Gamma} M_{\alpha}$ onto $\prod_{\alpha \in \Gamma} M_{\alpha}$ is universal. Thus $\prod_{\alpha \in \Gamma} M_{\alpha}$ has the fpp.

Lemma 2. Suppose that $\left\{f_{\alpha}: X_{\alpha} \rightarrow M_{\alpha} \mid \alpha \in \Gamma\right\}$ is a family of surjective mappings of continua. If

$$
\prod_{\alpha \in \Gamma} f_{\alpha}: \prod_{\alpha \in \Gamma} X_{\alpha} \rightarrow \prod_{\alpha \in \Gamma} M_{\alpha}
$$

is universal, then $f_{\alpha}$ is universal for each $\alpha \in \Gamma$.

Proof. Fix $\beta \in \Gamma$ and let $g_{\beta}: X_{\beta} \rightarrow M_{\beta}$ be a mapping. Define the mapping $g: \prod_{\alpha \in \Gamma} X_{\alpha} \rightarrow \prod_{\alpha \in \Gamma} M_{\alpha}$ by

$$
\pi_{\alpha} g(x)= \begin{cases}f_{\alpha} \pi_{\alpha}(x) & \text { for } \alpha \neq \beta \\ g_{\alpha} \pi_{\alpha}(x) & \text { for } \alpha=\beta\end{cases}
$$

By hypothesis, there exists an $x \in \prod_{\alpha \in \Gamma} X_{\alpha}$ such that $\prod_{\alpha \in \Gamma} f_{\alpha}(x)=g(x)$. It follows that $f_{\beta} \pi_{\beta}(x)=g_{\beta} \pi_{\beta}(x)$. Hence, $\pi_{\beta}(x)$ is a coincidence for the maps $f_{\beta}$ and $g_{\beta}$. 
Theorem 2. Let $\left\{M_{\alpha} \mid \alpha \in \Gamma\right\}$ be a family of continua. For the product of arbitrary surjective maps of continua to the $M_{\alpha}$ 's to be a universal map, it is necessary and sufficient that $\sigma_{0}^{*}\left(M_{\alpha}\right)=0$ for each $\alpha \in \Gamma$.

Proof. Sufficiency follows from Corollary 1. Necessity follows from Lemma 2 and the fact that $\sigma_{0}^{*}\left(M_{\alpha}\right)=0$ if and only if each surjective map of a continuum onto $M_{\alpha}$ is universal.

The author wishes to thank Charles Hagopian and Eldon Vought for suggestions which led to the improvement of this paper.

\section{REFERENCES}

[1] R. F. Brown, The Fixed Point Property and Cartesian Products, Amer. Math. Monthly 89 (1982), no. 9, 654-678. MR 84e:54047

[2] J. Bustamante, R. Escobedo, and F. Macías-Romero, A Fixed Point Theorem for Whitney Blocks, Topology Appl. 125 (2002), 315-321. MR 2003i:54038

[3] H. Cook, W. T. Ingram, and A. Lelek, Eleven Annotated Problems about Continua, Open Problems in Topology, J. van Mill and G. M. Reed, Editors, North-Holland, Amsterdam, 1990, pp. 295-302.

[4] J. F. Davis, The Equivalence of Zero Span and Zero Semispan, Proc. Amer. Math. Soc. 90 (1984), 133-138. MR 85k:54036

[5] E. Dyer, A Fixed Point Theorem, Proc. Amer. Math. Soc. 7 (1956), 662-672. MR 17:1232d

[6] R. Escobedo, M. de J. López, and S. Macías, On the Hyperspace Suspension of a Continuum, preprint.

[7] W. Holsztyński, Une Généralisation du Théorème de Brouwer sur les Points Invariants, Bull. Acad. Polon. Sci. Sér. Sci. Math. Astronom. Phys. 12 (1964), no. 10, 603-606. MR 30:4248

[8] _ Universality of Mappings onto the Products of Snake-like Spaces. Relation with Dimension, Bull. Acad. Polon. Sci. Sér. Sci. Math. Astronom. Phys. 16 (1968), no. 3, 161167. MR 37:5857

[9] S. Husseini, The Product of Manifolds with the fpp need not have the fpp, Amer. J. Math. 99 (1977), 919-931. MR 56:13203

[10] K. Kuratowski, Topology, Vol. II, Academic Press, New York, 1968. MR 41:4467

[11] W. Lopez, An Example in the Fixed Point Theory of Polyhedra, Bull. Amer. Math. Soc. 73 (1967), 922-924. MR 35:7323

[12] M. M. Marsh, Some Generalizations of Universal Mappings, Rocky Mountain J. Math. 27 (1997), no. 4, 1187-1198. MR 99d:54027

[13] , s-Connected Spaces and the Fixed Point Property, Topology Proc. 8 (1983), 85-97. MR 85f:54101

[14] R. L. Moore, Foundations of Point Set Theory, Amer. Math. Soc. Colloquium Publications, Vol. XIII, Providence, RI, 1962. MR 27:709

[15] L. G. Oversteegen and E. D. Tymchatyn, On Span and Weakly Chainable Continua, Fund. Math. 122 (1984), 159-174. MR 85m:54034

[16] R. H. Sorgenfrey, Concentrating Triodic Continua, Amer. J. Math. 66 (1944), 439-460. MR 6:96d

Department of Mathematics and Statistics, California State University, Sacramento, SaCramento, California 95819-6051

E-mail address: mmarsh@csus.edu 